\title{
UMA ESTIMATIVA DA CONTRIBUIÇÃO TARIFÁRIA PARA O EFEITO-FRONTEIRA NO BRASIL*
}

\author{
Orlando Monteiro da Silva ${ }^{\dagger}$ \\ Fernanda Maria de Almeida $\ddagger$
}

\begin{abstract}
Resumo
O objetivo desse trabalho foi analisar, explicitamente, o efeito das tarifas e das barreiras não-tarifárias (BNTs) nas exportações brasileiras e sua contribuição para o efeito-fronteira. Utilizou-se um modelo de gravidade e um procedimento para calcular o equivalente tarifário das barreiras nãotarifárias. No modelo mais simples, o efeito-fronteira estimado mostrou que os estados brasileiros comercializam, em média, 33 vezes mais entre si do que com o resto do mundo. A introdução explícita da tarifa no modelo indicou um efeito redutor nas exportações, mais que proporcional ao aumento tarifário. Isolando a influência das tarifas do efeito-fronteira, os estados brasileiros ainda comercializariam 26 vezes mais entre si. O cálculo do equivalente tarifário das barreiras não-tarifárias mostrou que as BNTs correspondiam à uma tarifa ad valorem de aproximadamente $29 \%$.
\end{abstract}

Palavras-chave: barreiras não-tarifárias, efeito fronteira, modelo de gravidade

\begin{abstract}
The objective of this paper was to analyze, explicitly, the effect of tariffs and non-tariff barriers (NTBs) on the Brazilian exports and its contribution to the border effect. It was used a gravity model with a procedure to calculate the tariff equivalent of non-tariff barriers. In the simplest model, estimated border effect showed that Brazilian states trade on average 33 times more among themselves than with the rest of the world. Explicit introduction of tariffs in the model showed that exports suffered a greater reduction than the increase on tariffs. The isolation of the influence of tariffs on the border-effect indicated that Brazilian states would still trade 26 times more among themselves. The calculated tariff equivalent of non-tariff barriers showed that those NTBs correspond to a tariff ad valorem of approximately $29 \%$.
\end{abstract}

Palavras-chave: Tariffs, non-tariff barriers, border effect, gravity model JEL classification: F10, F14, F15

\footnotetext{
* Pesquisa realizada com apoio do CNPq

† Professor do Departamento de Economia - Universidade Federal de Viçcosa. Email: odasilva@ufv.br

‡ Doutoranda em Economia Aplicada no Departamento de Economia Rural - Universidade Federal de Viçcosa.f_m_almeida@yahoo.com.br
} 


\section{Introdução}

A literatura recente sobre a economia internacional tem dado grande atenção à importância das fronteiras nacionais para o comércio entre as nações. Esse retorno ao tema, que foi iniciado a partir do trabalho de McCallum (1995), evidenciou que, em 1998, o comércio entre as províncias canadenses era em torno de 22 vezes maior do que o comércio com os estados dos Estados Unidos. Esse viés para as compras domésticas refletiria uma medida do grau em que os mercados são segmentados pelas fronteiras políticas. Apesar das muitas razões para a sua existência (barreiras comerciais: tarifárias e não-tarifárias; naturais: custo e tempo de transporte; culturais e institucionais; a exposição ao risco cambial; e preferências por produtos domésticos, etc.), o resultado de McCallum despertou grande interesse pela sua magnitude e, principalmente, por acontecer entre dois países que são grandes parceiros comerciais. Diversos autores utilizaram o mesmo modelo proposto para aquele estudo, repetindo a análise para os mesmos países (Helliwell 1996, Anderson e Van Wincoop 2003), ou para países diferentes (Weil 1996, Feenstra et al. 1998, Redding e Venables 2000), sempre encontrando estimativas que indicavam um viés significativo para os bens domésticos em detrimento dos bens importados.

No Brasil, Hidalgo e Vergolino (1998) analisaram o comércio do Nordeste com as demais regiões e o exterior, utilizando dados de 1991. Esses autores estimaram um efeito-fronteira de 1,5 para o Nordeste e de 11 para o Brasil, refletindo as preferências em excesso pelo comércio local, em comparação com o comércio das demais regiões do Brasil e do mercado internacional, respectivamente. Silva et al. (2004) atualizaram a mesma análise, com dados de 1999, considerando também o comércio intra-estados do Nordeste e encontraram resultados muito similares aos do trabalho de Hidalgo e Vergolino: 7,4 para o Nordeste (não-significativo) e 12,2 para o Brasil.

Paz (2003) utilizou dados de corte seccional dos 26 estados brasileiros e 192 países, empilhados dos anos de 1991, 1997, 1998 e 1999. Ele estimou equações de uma subamostra de 75 países e de um total de 192 países, nas quais considerava ou não as observações zero. Os resultados foram muito divergentes. Considerando as observações zero da amostra, os valores estimados do efeito-fronteira foram exageradamente elevados. Quando as observações zero foram descartadas, o menor valor encontrado foi igual a 15, indicando que o comércio interestadual é 15 vezes maior do que o internacional.

Daumal e Zignago (2005) utilizaram o modelo de gravidade para estimar o efeito-fronteira e inferir sobre o grau de integração entre os estados brasileiros e o nível de engajamento do país no mercado internacional. Os resultados indicaram que o comércio intranacional brasileiro é 33 vezes maior que o comércio internacional e que o efeito-fronteira médio dos estados do Brasil aumentou de 315 em 1991 para 370 em 1999, apontando uma integração imperfeita e limitada dos estados ao mercado global.

O mesmo resultado de Daumal e Zignago, quanto ao efeito-fronteira brasileiro, foi encontrado por Silva et al. (2007), para o ano de 1999. Esses autores utilizaram diversas formulações para o modelo, com dados do comércio entre os estados, e dos 40 principais parceiros comerciais do Brasil, obtendo um efeito-fronteira médio em torno de 33. Eles mostraram também que estados com rendas similares tendem a comercializar mais entre si (Hipótese de Linder) e estados adjacentes comercializavam 3,7 vezes mais do que aqueles que não o eram. 
Com o objetivo de avaliar o grau de acesso a mercados das exportações industriais do MERCOSUL, Castilho (2005) estimou o efeito-fronteira com uma amostra composta por três países exportadores (Brasil, Argentina e Uruguai) e 75 importadores, nos anos de 1990 a 2000. Os resultados indicaram que o comércio intranacional era 104 vezes maior do que aquele entre os países membros e que o comércio com terceiros países era ainda menor (246 vezes), já descontado o efeito-tarifa. Apesar dos valores elevados do efeito-fronteira, Castilho mostrou que a diferença entre os comércios do tipo intra e internacional no MERCOSUL diminuiu ao longo da década, com a evolução dos coeficientes atestando que a integração regional foi maior do que a integração com terceiros países.

Em todos os estudos anteriormente mencionados, os autores ressaltaram a importância do aprofundamento da análise para o Brasil (Paz 2003, Castilho 2005, Daumal e Zignago 2005), sugerindo a consideração de formas funcionais diferentes e de variáveis que refletissem características específicas do país (Paz 2003, Castilho 2005, Silva et al. 2007) e que permitissem melhor compreensão da magnitude e das causas do efeito-fronteira.

Visando contribuir para um melhor entendimento do efeito-fronteira no Brasil, o objetivo deste estudo é utilizar a fórmula básica do modelo de gravidade, expandindo-a com variáveis específicas para o caso brasileiro, incluindo explicitamente tarifas e barreiras não-tarifárias (BNT). Dessa forma, pretendese, ainda, calcular o equivalente tarifário das barreiras não-tarifárias nas exportações brasileiras.

\section{Metodologia}

Uma abordagem típica da análise dos fluxos de produtos no comércio internacional é o emprego dos modelos de gravidade. Os modelos de gravidade baseiam-se na fórmula da Lei da Gravidade de Newton, que afirma que a força de atração entre dois objetos quaisquer $i$ e $j$ depende diretamente de suas massas $\left(M_{i}\right.$ e $\left.M_{j}\right)$ e inversamente do quadrado da distância entre eles $\left(D_{i j}\right)^{2}$, podendo ser expressa como: $F_{i j}=G\left(M_{i} * M_{j}\right) /\left(D_{i j}\right)^{2}$, em que $G$ é a constante de gravidade.

Na década de 1960, economistas (Tinbergen 1962, Linnemann 1966) descobriram que a fórmula $F i j=G(M i a * M j b) /(D i j) 2$ servia bem para explicar o comércio entre duas regiões; se $F_{i j}$ fosse o fluxo de bens da região $i$ para a região $j ; M_{i}$ e $M_{j}$ fossem o tamanho econômico relativo de cada região; $D_{i j}$ fosse a distância entre elas; e $G, a$ e $b$ fossem constantes relacionadas à forma funcional da relação econômica.

A falta de fundamentação teórica inicial foi suprida por diversos desenvolvimentos que começaram, em 1979, com o modelo de substitutabilidade imperfeita dos bens, de Anderson (1979); os de competição monopolística, de Bergstrand (1989) e Deardoff e Stern (1997); e, principalmente, os de Anderson e Van Wincoop (2003), que consideraram os bens diferenciados por local de origem, utilizando uma função de elasticidade de substituição constante (CES). Atualmente, fala-se em "excesso" de fundamentação teórica Azevedo et al. (2006), o que tem renovado o interesse pelo modelo. Uma boa resenha pode ser encontrada no trabalho de Paz (2003).

Sua primeira utilização para estimar o efeito-fronteira se deve a McCallum (1995), que mostrou que, em 1998, o comércio entre duas províncias canaden- 
ses era em torno de 22 vezes maior que o comércio dessas províncias com os Estados Unidos. No modelo proposto por McCallun, o efeito-fronteira foi estimado, introduzindo-se no modelo padrão de gravidade uma variável dummy, que assumiu o valor 1 para o comércio entre as províncias do Canadá, e zero para o comércio entre as províncias e os Estados Unidos.

Na forma log-linear, o modelo básico de McCallun pode ser expresso como:

$$
\ln X_{i j}=\beta_{0}+\beta_{1} \ln P I B_{i}+\beta_{2} \ln P I B_{j}+\beta_{3} \ln D_{i j}+\beta_{4} B P+\mu_{i j}
$$

em que $X_{i j}=$ exportações do estado $i$ para o estado ou país $\mathrm{j} ;$ PIB $B_{k}=$ PIB nominal dos estados ou países $i$ e $j ; D_{i j}=$ distância em $\mathrm{km}$ do estado $i$ ao estado ou país $j ; B P=$ variável dummy que assume o valor 1 se as exportações do estado $i$ vão para o estado $j$ do país de origem e zero se elas têm como destino outro país; $\beta_{0}$ a $\beta_{4}=$ parâmetros com sinais positivos, à exceção de $\beta_{3}$; e $\mu=$ o termo de erro.

Depois desse estudo, diversos outros foram realizados, incluindo variáveis explicativas como proximidade geográfica (adjacência), ou distância relativa (remoteness), população, língua comum, ou laços culturais ou, ainda, variáveis dummies para captar relações preferenciais de comércio.

Anderson e Van Wincoop (2003) argumentaram, contudo, que na forma especificada o modelo tende a superestimar o efeito-fronteira, por sofrer do problema da omissão de variáveis. Um modelo correto deveria considerar, desde o início, preços diferentes entre os países, decorrentes dos custos de transporte e das barreiras. Portanto, eles modificaram a equação, introduzindo variáveis de "resistência multilateral" que indicavam quanto do comércio de um país com determinado parceiro seria aumentado pela resistência que esses países tinham em comercializar com todos os demais países. Para tanto, eles assumiram a diferenciação dos bens por local de origem com os consumidores de determinado país, maximizando a seguinte função de utilidade CES:

$$
\max \left(\sum \beta_{j}^{\frac{1-\sigma}{\sigma}} c_{i j}^{\frac{\sigma-1}{\sigma}}\right)^{\frac{\sigma}{\sigma-1}} \text {, sujeito a } \sum p_{i j} c_{i j}=y_{i}
$$

em que $c_{i j}$ é o consumo dos bens do país $j$ pelos consumidores do país $i, \sigma$ é a elasticidade de substituição entre todos os bens, $\beta_{i}$ é um parâmetro de distribuição positivo, $y_{j}$ é a renda nominal dos residentes do país $j$ e $p_{i j}$ é o preço dos bens do país $i$ para os consumidores do país $j$. A solução desse problema daria uma equação de gravidade, na forma:

$$
X_{i j}=\left(\frac{Y_{j} * Y_{j}}{Y^{w}}\right)\left(\frac{t_{i j}}{P_{i} * P_{j}}\right)^{1-\sigma},
$$

com $X_{i j}$ indicando as exportações do país ou região $i$ para o país ou região $j$; $Y_{i}, Y_{j}$ e $Y_{w}$, as rendas nominais dos países $i$, $j$ e do mundo, respectivamente; $t_{i j}$, os custos do comércio entre $i$ e $j$; e $P_{i}$ e $P_{j}$, os índices de preço ou de resistência multilateral dos países.

Essa equação evidencia que, após controlar para o "tamanho" dos países, o comércio entre eles vai depender das barreiras bilaterais entre $i$ e $j$, relativo à barreira média que eles enfrentam no tocante a todos os demais parceiros.

Anderson e Van Wincoop (2003) sugeriram a seguinte forma funcional, 
considerando uma elasticidade-renda unitária:

$$
\begin{aligned}
\ln \left(\frac{X_{i j}}{Y_{i} Y_{j}}\right)=\beta_{0}+(1-\sigma) \rho \ln D_{i j}+(1-\sigma) & \ln B_{i j} \\
& +(1-\sigma) \ln P_{i}+(1-\sigma) \ln P_{j}+\mu_{i j},
\end{aligned}
$$

com $\rho$ representando uma constante exponencial da variável distância e as demais variáveis definidas como anteriormente. Na realidade, a diferença básica entre essa equação e aquela estimada por McCallun são os dois índices de preços. Contudo, como os valores de $P_{i}$ e $P_{j}$ não são diretamente observados, podem-se utilizar índices de preços aos consumidores, como proxies para os índices de resistência, como fizeram Baier e Bergstrand (2001); utilizar valores estimados, como fizeram Anderson e Van Wincoop (2003); ou, ainda, fazer uso dos efeitos fixos, como fizeram Redding e Venables (2000). Feenstra (2004) argumentou que a utilização do efeito fixo é a mais adequada pela simplicidade computacional, além de fornecer estimativas consistentes do efeito-fronteira.

É importante ressaltar que os índices de resistência multilaterais têm relação direta com as barreiras bilaterais ao comércio e que desconsiderá-los traria problemas de viés pela omissão de variáveis.

Para este estudo, propõe-se a utilização direta e simultânea das tarifas e das barreiras não-tarifárias nas equações estimadas, como proposto pela Conferência das Nações Unidas sobre Comércio e Desenvolvimento - UNCTAD (2008), permitindo obter a equivalência tarifária das barreiras não-tarifárias, com base nos fluxos de comércio observados.

O modelo a ser estimado tem, portanto, a seguinte forma funcional:

$$
\begin{aligned}
\ln X_{i j}=\beta_{0}+\beta_{1} \ln P I B_{i}+\beta_{2} \ln P I B_{j}+\beta_{3} \ln D_{i j}+\beta_{4} \ln \left(1+\tau_{i j}\right) \\
+\beta_{5} Q_{i j}+\beta_{6} E_{i}+\beta_{7} A D J_{i j}+\mu_{i j},
\end{aligned}
$$

$X_{i j}=$ as exportações do estado i para o estado ou país $j ; P I B_{k}=$ o PIB nominal dos estados ou países $i$ e $j$; $D_{i j}=$ distância em $\mathrm{km}$ do estado $i$ ao estado ou país $j ; \tau_{i j}=$ tarifa imposta pelo país $j$ ao produto em análise do estado $i$; $Q_{i j}=$ barreira não-tarifária imposta pelo país $j$ ao produto em análise do estado $i ; E_{i}=$ variável dummy que assume o valor 1 quando o estado $i$ exportar para outro estado e zero quando o estado $i$ exportar para outro país; $A D J_{i j}$ $=$ variável dummy que assume o valor 1 quando o estado $i$ exportar para um estado ou país com os quais tenha fronteira comum e zero caso contrário; $\beta_{0}$ 's $=$ parâmetros com sinais positivos, à exceção de $\beta_{3}, \beta_{4}$ e $\beta_{5} \mu_{i j}=0$ termo de erro.

As estimativas da equação anterior podem ser utilizadas para se obterem os equivalentes tarifários das barreiras não-tarifárias. Considere $Z_{i j n t}$ como as demais variáveis explicativas que não $Q_{i j t}$, cuja soma dos coeficientes pode ser escrita como:

$$
\ln X_{i j}=\sum_{n \neq 5} \beta_{n} Z_{n i j}+\beta_{5} Q_{i j}
$$

Note que $Q_{i j}$ é igual a 1 se uma barreira não-tarifária existe e zero caso contrário. Assim, a diferença prevista no fluxo de comércio entre um par de países com a barreira não-tarifária e o mesmo par de países sem a barreira seria: 


$$
\begin{aligned}
\ln X_{i j, \mathrm{com} \mathrm{BNT}}-\ln X_{i j, \mathrm{sem} \mathrm{BNT}}=\sum_{n \neq 5} \beta_{n} Z_{n i j}+ & \beta_{5}(1) \\
& -\left(\sum_{n \neq 5} \beta_{n} Z_{n i j}+\beta_{5}(0)\right)=\beta_{5}
\end{aligned}
$$

O mesmo procedimento pode ser utilizado para captar o efeito da imposição de uma tarifa, comparado com a inexistência de tarifa no comércio entre cada par de países.

$$
\begin{aligned}
\ln X_{i j, \mathrm{com} \text { tarifa }}-\ln X_{i j, \text { sem tarifa }}=\sum_{n \neq 4} \beta_{n} Z_{n i j}+\beta_{4} \ln \left(1+\tau_{i j}\right) & \\
& -\sum_{n \neq 4} \beta_{n} Z_{n i j}+\beta_{4}(1)=\beta_{4} \ln \left(1+\tau_{i j}\right)
\end{aligned}
$$

O equivalente tarifário de uma barreira não-tarifária seria aquela tarifa que tem o mesmo impacto no fluxo de comércio, sendo obtida por meio da igualdade das expressões (7) e (8):

$$
\beta_{4} \ln \left(1+\tau_{i j}\right)=\beta_{5} \quad \text { ou, } \quad \tau=\exp \left(\frac{\beta_{5}}{\beta_{4}}\right)-1,
$$

que fornece a tarifa ad valorem equivalente àquela barreira não-tarifária.

Os dados utilizados são do ano de 1999. As observações sobre as exportações dos 26 estados brasileiros e o Distrito Federal, uns para os outros e para os principais parceiros (76 países) comerciais, foram obtidos de Vasconcelos e Oliveira (2006) e do site Aliceweb, do Ministério do Desenvolvimento, Indústria e Comércio Exterior (MDIC). A escolha dos 76 países se deu em função da existência de fluxos de comércio com os estados naquele ano. Ao fazer essa opção, reduziu-se o número de observações zero da amostra, que passaram a existir somente no comércio entre alguns dos estados. Dados sobre o PIB e a população (nacional e por unidade da federação) foram obtidos do Instituto Brasileiro de Geografia e Estatística (IBGE), enquanto os dados dos países selecionados o foram do Penn World Table. Todos os valores monetários eram nominais e foram expressos em reais. Os PIBs dos demais países foram transformados em reais, utilizando-se a taxa de câmbio média (reais/US\$), para o ano de 1999. As distâncias, medidas em km, foram obtidas do site Areaseg.com e representam as distâncias físicas entre as capitais de cada estado. Para os demais países, considerou-se a distância dos estados até Brasília e de lá até a capital de cada um dos países, sendo esses dados retirados do Centre d'Etudes Prospectives et d'Informations Internationales (CEPII). Os valores das tarifas e das barreiras não-tarifárias foram obtidos do trabalho de Anderson e Van Wincoop (2004).

\section{Resultados}

Antes de apresentar os resultados obtidos da estimação do modelo proposto, torna-se importante descrever as maneiras pelas quais tarifas e barreiras nãotarifárias foram coletadas e disponibilizadas para análise. 
Os valores das tarifas considerados por Anderson e Van Wincoop (2004) se referem à média aritmética das muitas linhas tarifárias, em cada país, obtidas do site TRAINS da UNCTAD. O pequeno número de países considerados por esses autores (49 mais a União Européia) foi justificado pelas dificuldades típicas de coleta do programa (TRAINS) no ano de análise. Desses países, 48 (33 mais 15 da União Européia) comercializaram com o Brasil no ano de 1999 e tiveram suas taxas tarifárias consideradas. De maneira geral, os dados indicaram que nos países desenvolvidos os valores das tarifas eram baixos (abaixo de $5 \%$ ) e relativamente altos nos países em desenvolvimento (acima de 10\%). Hong Kong e Suíça apresentaram tarifa zero e os Estados Unidos, uma tarifa de 1,9\%. As tarifas da Índia e de Bangladesh eram de 30 e 22,7\%, respectivamente. Para a estimação das equações na forma logarítmica, adotou-se a expressão $(1+\tau)$, com $\tau$ indicando a tarifa ad valorem para cada uma das nações consideradas. Os países não considerados representaram apenas $6 \%$ de todo o comércio internacional realizado pelo Brasil naquele ano.

Os dados sobre as barreiras não-tarifárias, também obtidos por Anderson e Van Wincoop (2004) do banco de dados TRAINS, dizem respeito a somente 34 países, refletindo as limitações maiores dessas informações. Os valores apresentados referem-se à taxa de cobertura ou à porcentagem das linhas tarifárias sujeitas às BNTs, em seu sentido mais amplo, ou seja, qualquer BNT relacionada a preços e quantidades (medidas compensatórias e anti-dumping), qualidade (normas, padrões, licenças etc.), além de embargos e proibições. A incidência de barreiras não-tarifárias nas diversas linhas tarifárias foi ponderada pelo volume de comércio e indicou a importância dessas medidas para os países em desenvolvimento. Argentina e Tunísia, por exemplo, tinham 76\% e $60 \%$ de suas linhas tarifárias cobertas por algum tipo de BNT, enquanto Líbano, Indonésia e Arábia Saudita estavam entre os países que não as adotaram no ano de 1999. Os 15 países da União Européia foram considerados separadamente, e, dos 33 países restantes na amostra, 26 apresentaram algum tipo de BNT. A incidência de BNTs em cada país foi introduzida no modelo por meio de uma variável dummy, que assumiu valores iguais a 1 ou zero, para índices de cobertura (BNT) positivos ou zero, respectivamente.

Os resultados da estimação dos modelos propostos estão apresentados na Tabela 1. Nas colunas numeradas de (1) a (5), mostram-se os resultados quando a variável dependente é o fluxo das exportações do estado $i$ para o estado ou país $j$, enquanto os resultados das colunas de (6) a (10) indicam os resultados quando a variável dependente é expressa como a razão das exportações para o produto dos PIBs dos estados e/ou países. O grande número de observações utilizadas (1.334) proporcionou estabilidade às estimativas dos parâmetros. Não foi detectado nenhum caso de heterocedasticidade pelo teste de White, mas a autocorrelação esteve presente em todas as equações, com os resultados apresentados já corrigidos com relação ao problema.

Os coeficientes de determinação ajustados $\left(R^{2}\right)$ variaram entre 0,64 e 0,76. Em todos os casos, os coeficientes estimados das variáveis foram significativos ao nível de $1 \%$, e, especificamente, aqueles da equação de gravidade básica (PIBs e distância) apresentaram magnitudes condizentes com outros estudos dessa natureza. Como foram estimados na forma log-linear, esses coeficientes podem ser lidos diretamente como elasticidades. No caso da variável distância, por exemplo, pode-se dizer que, em média, aumentos de $10 \%$ na distância entre dois estados ou países reduziriam em 10\% o comércio entre eles. Esse resultado é coerente com o descrito por Head (2003), que conduziu uma meta- 
análise das estimativas das distâncias em 595 equações de gravidade, encontrando um valor médio igual a 0,94 . Vale ressaltar, contudo, a complexidade do coeficiente da variável distância, que capta não somente o efeito dos custos econômicos do transporte (custos do despacho, de sincronização, de comunicação e de transação), como também os custos da "distância cultural" entre os parceiros.

A variável dummy utilizada para diferenciar o comércio entre estados e países (efeito-fronteira) apresentou também coeficientes estáveis nos dois casos, variando entre 3,27 e 3,51 quando a variável dependente era expressa pelas exportações, e entre 4,32 e 4,55 quando essa variável dependente era expressa pela razão das exportações para o produto dos PIBs. A correlação simples entre essa última variável e a dummy utilizada para diferenciar o comércio entre estados daquele com os demais países foi muito maior $(0,83)$ do que aquele calculado entre as exportações e a dummy. De maneira geral, o produto dos PIBs estaduais foi menor do que o produto dos PIBs entre estados e países, levando à maior associação entre a variável dummy para comércio entre estados e a razão das exportações e o produto dos PIBs.

Como efeito-fronteira, no caso brasileiro foram considerados os resultados dos modelos nos quais a variável dependente é o fluxo das exportações, equações (2) a (5), que são coerentes com aqueles encontrados por Daumal e Zignago (2005) e Silva et al. (2007). Dessa forma, pode-se dizer que os estados brasileiros comercializam entre si 26 e 33 vezes (exp. do coeficiente da variável dummy) mais do que com outros países. Os valores encontrados para os coeficientes da variável dummy para diferenciar os estados e/ou países adjacentes permitem inferir que estados e/ou países com fronteira comum comercializam de 2,4 a 3,1 vezes mais entre si.

Nos dois modelos estimados, a consideração explícita das variáveis tarifas e barreiras não-tarifárias melhorou os resultados. Os coeficientes estimados dessas variáveis foram sempre significativos e com sinais coerentes. Os resultados indicam que uma redução de $10 \%$ nas tarifas ad valorem pelos países com os quais o Brasil tem relações comerciais aumentaria o comércio entre eles entre 27 e 33\%. As barreiras não-tarifárias também apresentaram coeficientes significativos e reforçam os efeitos das tarifas. Rodrik (1995) já tinha mostrado que as barreiras tarifárias e não-tarifárias são complementares quando estimadas em modelos de equação única de seção cruzada, mas que são substitutas quando as políticas comerciais são analisadas ao longo do tempo. Os coeficientes estimados das variáveis tarifas e barreiras nãotarifárias são, então, utilizados para obter o equivalente tarifário das BNTs.

A utilização dos coeficientes das variáveis tarifas e barreiras não-tarifárias obtidas pela equação (5) na expressão (9), da metodologia, permite inferir que o equivalente tarifário das barreiras não-tarifárias é de $28,7 \%$, ou seja, o preço de acesso das exportações brasileiras nos mercados considerados é acrescido em quase $29 \%$ pela ação do conjunto das BNTs. O mesmo método utilizado na equação (9) evidencia um equivalente tarifário das BNTs de $49,7 \%$. Esse último resultado é aumentado pela mesma razão que aumentou o efeito-fronteira quando o modelo teve a variável dependente especificada como a razão das exportações sobre o produto dos PIBs. Deve ser observado que, ao agregar todo o comércio de um país, perde-se quanto à especificidade dos setores, mas se permite a quantificação do efeito que as diversas formas de barreiras não-tarifárias têm sobre o comércio de um país.

Também, como a variável exportação é um dos componentes do PIB, existe 


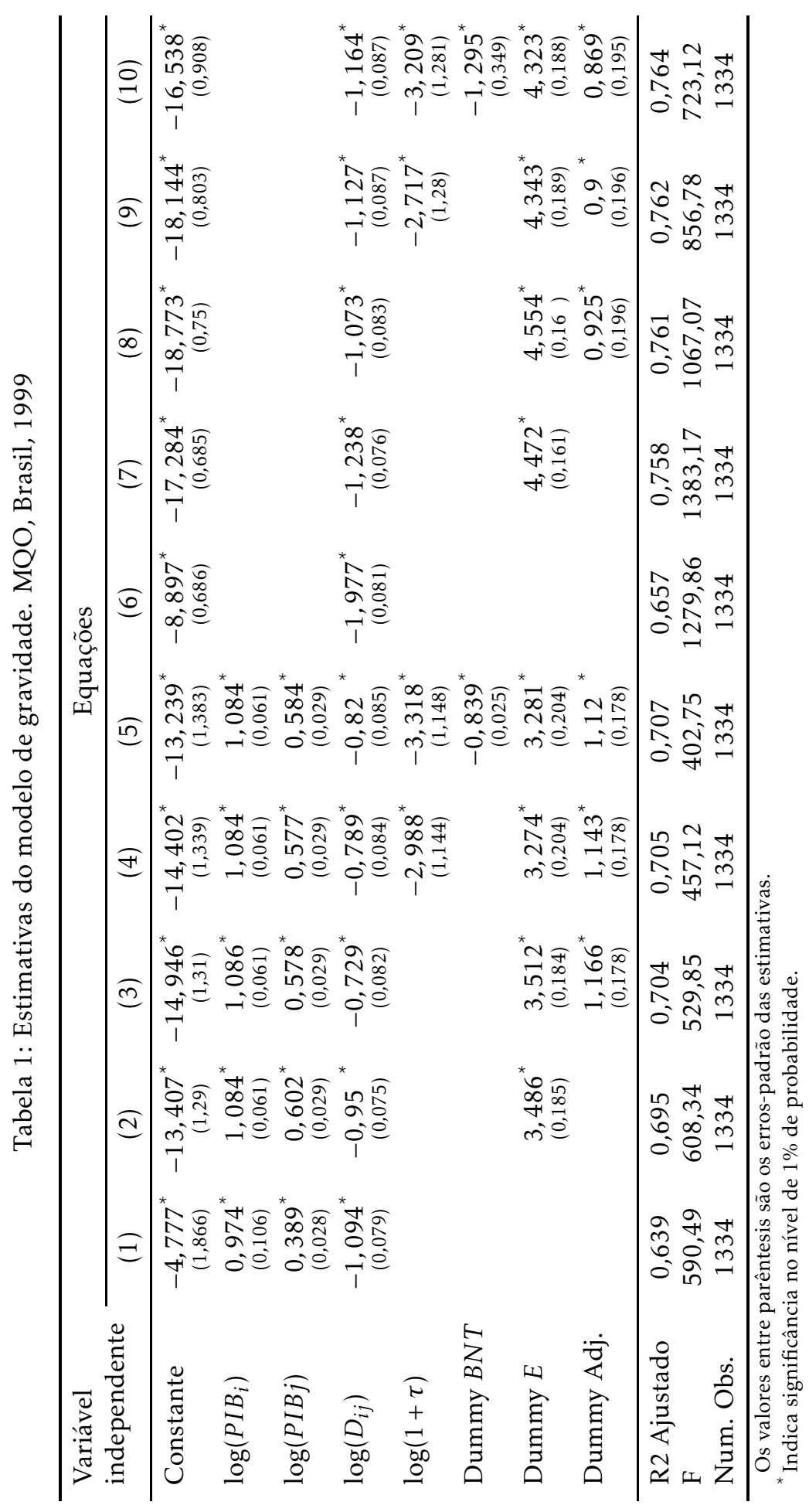


a possibilidade de endogeneidade entre essas variáveis e da elevação do coeficiente estimado para a variável PIB. As opções de correção desse problema seriam as utilizações de variáveis instrumentais para a variável PIB (que usualmente também são correlacionados com os fluxos de comércio), ou a utilização de séries temporais e de defasagem do PIB, o que é impossível nesse caso pela inexistência das séries sobre os fluxos de comércio.

\section{Conclusões}

O objetivo deste trabalho foi analisar, explicitamente, o efeito das tarifas e das barreiras não-tarifárias nas exportações brasileiras e sua contribuição para o efeito-fronteira. Nos modelos adotados consideraram-se as exportações entre todos os 26 estados brasileiros mais o Distrito Federal e as exportações destes para 76 diferentes países, com resultados bastante coerentes com os de estudos similares.

No modelo mais simples, o efeito-fronteira estimado indicou que os estados brasileiros comercializam, em média, 33 vezes mais entre si do que com o resto do mundo. A introdução explícita da tarifa no modelo indicou um efeito redutor nas exportações mais que proporcional ao aumento tarifário e uma queda no efeito-fronteira. Isolando a influência das tarifas desse efeito, os estados brasileiros ainda comercializariam 26 vezes mais entre si do que com o resto do mundo. Essa queda em relação ao valor representa a contribuição tarifária do efeito-fronteira que engloba todos os fatores que fazem que os estados dêem preferência ao comércio doméstico sobre o internacional.

A consideração explícita da variável barreira não-tarifária no modelo estimado não causou alterações diretas no efeito-fronteira, mas aumentou o efeito das tarifas, o que comprova sua complementaridade. O cálculo do equivalente tarifário das barreiras não-tarifárias indicou, no entanto, um valor expressivo, sugerindo que, nas suas diversas formas, as BNTs aplicadas às exportações brasileiras correspondiam a uma tarifa ad valorem de aproximadamente $29 \%$.

É importante chamar a atenção para a forma pela qual as tarifas foram mensuradas e os problemas de agregação a ela relacionados. Coletadas nos níveis de linha tarifária (6 e 8 dígitos do sistema harmonizado), cada país apresenta centenas ou milhares de tarifas diferentes. Sua utilização como média aritmética simples certamente resulta na perda de informações importantes sobre os valores extremos das tarifas e os diferentes pesos de cada produto no fluxo comercial total. A alternativa, que seria a ponderação das tarifas pelo peso dos produtos, também é criticada, por minimizar os efeitos das tarifas altas. Assim, ao utilizar os resultados deste estudo, deve-se considerar esse possível viés da agregação.

O método de análise utilizado parece, contudo, bastante promissor para a análise de fluxos de comércio setoriais, em que a relevância das tarifas é maior e os problemas de agregação são minimizados.

\section{Referências Bibliográficas}

J. A. Anderson e E. Van Wincoop. Gravity with gravitas: a solution to a border puzzle. American Economic Review, 93:170-192, 2003.

J. A. Anderson e E. Van Wincoop. Trade costs. Journal of Economic Literature, 42:691-751, 2004. 
J. E. Anderson. A theoretical foundation fir the gravity equation. American Economic Review, 1:106-116, 1979.

A. F. Azevedo, M. S. Portugal, e P. C. F. Barcellos Neto. Impactos comerciais da área de livre comércio das américas: uma aplicação do modelo gravitacional. Revista de Economia Contemporânea, 10:237-267, 2006.

S. Baier e J. H. Bergstrand. The growth of world trade: tariffs, transport costs and income similarity. Journal of Internatioanl Economics, 53:1-27, 2001.

J. H. Bergstrand. The generalized gravity equation, monopolistic competition, and the factor proportion theory in international trade. Review of Economics and Statistics, 71:43-153, 1989.

M. R. Castilho. Integração regional e internacional do mercosul: uma medida de integração e de acesso a mercados a partir da estimação do. Technical report, ANPEC, http://www.anpec.org.be/encontro2005/artigos, 2005. URL http://www. anpec.org.be/encontro2005/artigos.

M. Daumal e S. Zignago. The border effects in brazil. Technical report, Université Paris Dauphine, 2005. URL http://www. dauphine.fr/globalisation/ daumal2.pdf.

A. V. Deardoff e R. M. Stern. Measurement of non-tariff barriers. Master's thesis, OCDE, 1997.

R. C. Feenstra. Advanced international trade: theory and evidence. Master's thesis, Princeton University, 2004.

R. C. Feenstra, J. R. Markusen, e A. K. Rosenblatt. Undestanting the home market effect and the gravity aquation: the role of differentiationg goods. Technical report, NBER, 1998.

K. Head. Gravity for beginners. Master's thesis, University of British Columbia, 2003.

J. F. Helliwell. How much do national borders matter? DC: Brookings Institution Press, 1996.

A. B. Hidalgo e J. R. Vergolino. O nordeste e o comércio inter-regional e internacional: um teste dos impactos por meio do modelo gravitacional. Economia Aplicada, 2:707-725, 1998.

H. Linnemann. An econometric study of international trade flows. Amsterdam: North Holland, 1966.

J. McCallum. National bosders matter: Canadá-u.s. regional trade patterns. American Economic Review, 85:615-623, 1995.

L. S. Paz. Brazilian international and inter-state trade flows: an exploratory analysis using the gravity model. Master's thesis, FGV/RJ, 2003.

S. Redding e A. J. Venables. Economic geography and international inequality center for economic and policy research, 2000.

D. Rodrik. Political economy of trade policy. In Handbook of international economics, volume vol. III. Elsevier Science, 1995. 
M. V. B. Silva, W. R. Justo, e A. M Magalhães. Comércio interestadual e internacional do brasil e do nordeste: uma abordagem do modelo gravitacional, 2004. URL http://www.bnb.gov.br/content/ETENE/Anais/docs/ 2004comerciointerestadual.pdf.

O. M. Silva, F. M Almeida, e B. M. Oliveira. Intra-national versus internacional trade in brazil: measuring the border effect. In XII Anual Conference: Western Hemispheric Integration in a Competitive Global Envitonment, 2007.

J. Tinbergen. Shaping the world: suggestions for an international economy policy. New York: Twentieth Century Fund, 1962.

UNCTAD. Training package on trade policy analysis. Technical report, UNCTAD, 2008.

J. R. Vasconcelos e M. A. Oliveira. Análise da matriz de fluxo do comércio interestadual no brasil - 1999. Technical report, IPEA, 2006.

S. J. Weil. Intra-national versus international trade: how stubborn are nations in global integration? Master's thesis, NBER, 1996. 